\title{
Chemo Proteomics, a Valuable Tool for Biomarker and Drug Discovery
}

\author{
Marjeta Urh* \\ Director of Research, Promega Corporation, 2800 Woods Hollow Road, Madison WI 53711, USA
}

*Corresponding author: Marjeta Urh, Director of Research, Promega Corporation, 2800 Woods Hollow Road, Madison WI 53711, USA, Tel: (608) 443-3502; E-mail: marjeta.urh@promega.com

Rec date: June 26, 2014; Acc date: June 27, 2014; Pub date: June 30, 2014

Copyright: () 2014 Marjeta U. This is an open-access article distributed under the terms of the Creative Commons Attribution License, which permits unrestricted use, distribution, and reproduction in any medium, provided the original author and source are credited.

\section{Editorial}

Discovery and development of new drugs and therapeutic agents is greatly aided by knowing the identity of proteins affected by small molecule drugs, the drug targets. Understanding the drug targets is the key factor in development and optimization of drug candidates for high efficacy and low toxicity. Currently, large majority of the small molecule drugs on the market affect a small fraction of potential drug targets of the human proteome. In recent estimates the number of druggable targets is significantly higher, possibly as much as one order of magnitude higher, than the current number of protein targets through which small molecule drugs mediate their effect [1]. With the advancements in genomics and molecular biology, drug development has, over last several decades, largely depended on assessing activity against purified recombinant proteins. However, due to complexities of the cellular environment, presence of different splice variants and posttranslational modifications of the target protein and abundance of other proteins, these in vitro experiments may not predict the effect of drugs in complex in vivo systems. Furthermore the "off targets" effects of small molecule drugs cannot be easily assessed with in vitro experiments. Thus, evaluating phenotypic effect of small molecules using complex, in vivo cellular or animal models, offers a valuable alternative for drug development. In this process known as phenotypic screening small molecules are selected based on their ability to elicit desired phenotype, and only after this stage is completed, the search for drug target begins. This approach has resulted in discovery of majority of first-in-class drugs with novel mechanisms of action [2]. Thus, drug discovery process is seeing a comeback of phenotypic screening. This comeback has been facilitated with development of new tools for discovery of targets for small molecules identified in phenotypic screen.

Recent progress in development of such tools is a result of multidisciplinary approach of integrating biology, chemistry, mass spectrometry and computational data analysis. This new area has been given the name chemo-proteomics. Chemo-proteomic approaches are now routinely used for deconvolution of drug targets, assessment of the "off target" effects and selectivity of small molecules, and duration of target occupancy. Key enabling factor of chemo-proteomics are recent developments in mass spectrometry in particular the labeling methodologies and quantitative mass spectrometry $[3,4]$.

Over the last several years many different chemo-proteomics approaches have been developed and they can be classified in to three major categories; affinity pull-down, Capture Compound Mass Spectrometry and activity-based protein profiling [5-7]. While these methodologies differ in their approaches and design, they all aim at unbiased determination of drug targets in a complex cellular environment.
The basic concept of affinity pull-down based chemo-proteomics combines elements of affinity chromatography and active site chemical labeling of enzymes. This approach enables identification of all proteins that may interact with a small molecule in the context of the whole proteome. The small molecules identified in phenotypic screen are modified to become two-functional probes, by appending a sorting moiety, such as biotin, onto the small molecule. Alternatively, the small molecule can be immobilized directly onto a surface [8]. The original small molecule part of the probe, serves as bait for selective binding to proteins. This moiety is also known as selectivity function. The sorting molecule is used for affinity based isolation of probe-protein complex followed by mass spectrometry based identification of isolated proteins. The success of experiments and capture largely depends on design of the probes and appropriate selection of sites on small molecules that can withstand modification with sorting moiety without affecting the functionality. Coupling of the sorting group may lead to a change in activity, potency and solubility and multiple attachment sites may need to be exploited in absence of clear understanding of structure-activity relationship. Linker molecule which is used to connect sorting molecule onto the selectivity group also plays a critical role in the success of this approach, and it has to be properly optimized. Ideally, the probe should be tested for ability to generate the same phenotype as the original small molecule with similar potency. Limited permeability of modified molecules may interfere with this evaluation. Another important aspect that impacts the success of these experiments is evaluation of specificity of binding of isolated proteins to the probe used for capture. Frequently, nonspecifically bound proteins are also isolated in this process. The specificity of interactions is typically determined by competition experiments using the original non-modified small molecule to preblock the active site of specific targets. This prevents probe from binding to protein targets and isolation of these proteins in the process. Proteins absent from samples pre-treated with non-modified small molecule are likely candidates for specific protein targets of this molecule. In the approach where small molecule is attached directly onto a solid support, specificity of the interaction is evaluated by competitive elution of captured proteins using non-modified small molecule.

Interaction between probe and proteins in this approach is equilibrium based and is thus prone to dissociation based loses of target proteins, particularly the proteins with low affinity interactions. This is addressed by the second chemo-proteomics approach, the Capture Compound Mass Spectrometry [CCMS] [6]. Here, the probe consists of three elements, the selectivity function (the small molecule identified in phenotypic screen), an adjacent reactive group for crosslinking and a sorting function, such as biotin. The selectivity function and sorting moiety play a similar role as in affinity pull-down experiments described above. The reactive crosslinking group brings in a new functionality enabling covalent linkage of the probe to the 
Citation: Marjeta U (2014) Chemo Proteomics, a Valuable Tool for Biomarker and Drug Discovery. Mol Biol 3: e117. doi:

Page 2 of 2

bound protein and thus preventing equilibrium based losses of proteins during affinity purification. High selectivity and sensitivity of this approach has been successfully demonstrated with isolation of many protein classes including kinases and methyl transferases $[9,10]$. Optimization of the crosslinking moiety and crosslinking reaction is important for optimal efficiency and improved yields of captured proteins.

Third major category of chemo-proteomics approaches is affinity based protein profiling (ABPP) [7]. In the narrow-sense definition the ABPP probe comprises two elements. The first element is a selectivity group, which is designed to bind to the active site of the protein in covalent fashion. The second moiety is a functional reporter tag enabling visualization and enrichment. Ideally the ABPP probes would target a large number of proteins offering a tool for analysis of global functional state of the proteome. ABPP allows assessment of not only the abundance of particular protein or protein family to which selectivity group bind, but also evaluation of functional state of queried proteins in the cellular context. A competitive version, where small molecules are evaluated for their ability to block labeling of target proteins with the probe offers means to discover inhibitors of a particular class of target proteins.

Chemo-proteomics platform address fundamental challenges in drug discovery process. It provides means for profiling small moleculeprotein interaction and enables identification of small molecule protein targets in their endogenous state. This knowledge can then be used for target prioritization and drug development. Various chemoproteomics approaches have been further employed to discern mechanisms of action of small molecules including identification of the "off targets" which may positively or negatively affect effectiveness of small molecule drugs. One of the challenges that remain in this area is quantitative isolation of targets and development of new chemoproteomics methods enabling quantitation is desired.

\section{References}

1. Imming P, Sinning C, Meyer A (2006) Drugs, their targets and the nature and number of drug targets. Nat Rev Drug Discov 5: 821-834

2. Swinney DC, Anthony J (2011) How were new medicines discovered? Nat Rev Drug Discov 10: 507-519.

3. Patterson SD, Aebersold RH (2003) Proteomics: the first decade and beyond. Nat Genet 33 Suppl: 311-323.

4. Flory MR, Griffin TJ, Martin D, Aebersold R (2002) Advances in quantitative proteomics using stable isotope tags. Trends Biotechnol 20: S23-29.

5. Lenz T, Fischer JJ, Dreger M (2011) Probing small molecule-protein interactions: A new perspective for functional proteomics. J Proteomics 75: 100-115.

6. Köster H, Little DP, Luan P, Muller R, Siddiqi SM, et al. (2007) Capture compound mass spectrometry: a technology for the investigation of small molecule protein interactions. Assay Drug Dev Technol 5: 381-390.

7. Cravatt BF, Wright AT, Kozarich JW (2008) Activity-based protein profiling: from enzyme chemistry to proteomic chemistry. Annu Rev Biochem 77: 383-414.

8. Bantscheff M, Eberhard D, Abraham Y, Bastuck S, Boesche M, et al. (2007) Quantitative chemical proteomics reveals mechanisms of action of clinical ABL kinase inhibitors. Nat Biotechnol 25: 1035-1044.

9. Fischer JJ, Graebner Baessler OY, Dalhoff C, Michaelis S, Schrey AK, et al. (2010) Comprehensive identification of staurosporine-binding kinases in the hepatocyte cell line HepG2 using Capture Compound Mass Spectrometry (CCMS). J Proteome Res 9: 806-817.

10. Dalhoff C, Hüben M, Lenz T, Poot P, Nordhoff E, et al. (2010) Synthesis of S-adenosyl-L-homocysteine capture compounds for selective photoinduced isolation of methyltransferases. Chembiochem 11: 256-265. 